Édition spéciale - L'agrobusiness en temps d'effondrement planétaire: approches critiques Vol. 57, p. 258-269, jun. 2021. DOI: 10.5380/dma.v56i0.81394. e-ISSN 2176-9109

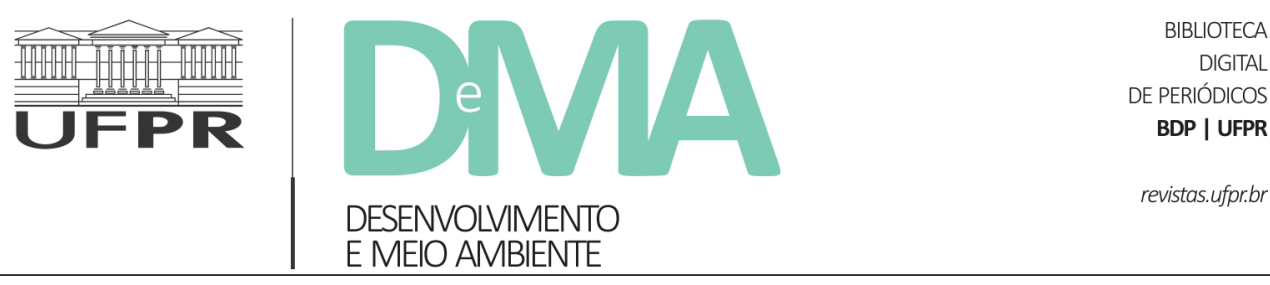

\title{
Un nouvel ordre juridique pour régénérer la Terre
}

\author{
Entretien $^{1}$ avec Valérie Cabanes ${ }^{2}$
}

RÉSUMÉ: Dans cet entretien, Valérie Cabanes - juriste active dans le domaine de la protection des droits humains (et de la nature) - commente sa trajectoire militante et ses liens actuels avec le mouvement qui a pour but d'inclure le crime d'écocide dans le champ du droit international. Son témoignage met en lumière les contradictions et les anomalies du modèle agro-industriel mis en œuvre au Brésil, dans une perspective qui prend en compte la complexité du débat sur les limites planétaires de la croissance des économies néolibérales.

1. En tant que juriste militante dans le domaine des droits de l'Homme, d'où vient votre intérêt pour la quête de nouvelles approches pour rendre l'écosystème Terre et les générations futures des "sujets de droit"? Dans quelle mesure la mobilisation de la notion de "crime d'écocide" peut-elle être justifiée et mobilisée pour rendre le droit international institué plus effectif face aux dérèglements et anomalies en cours?
J'ai pris conscience des failles du droit international concernant la protection de la nature et du climat en m'intéressant aux conflits engageant des peuples autochtones et des projets industriels au Québec puis au Brésil. J'ai démarré une thèse d'Anthropologie juridique en 2006 avec comme terrain de recherche pendant 3 ans le territoire et la culture des Innus d'Ekuanitshit en Minganie au Nord du Québec menacés par un projet de

\footnotetext{
${ }^{1}$ Entretien donné par e-mail le 28 avril 2021, à partir de questions posées par Paulo Freire Vieira et Marina Favrim Gasparini.

${ }^{2}$ Valérie Cabanes est juriste en droit international, spécialisée dans les droits de l'homme et le droit humanitaire. Après deux décennies dans des ONG de terrain œuvrant dans les champs de la solidarité internationale, elle oeuvre depuis 2013 à la reconnaissance du crime d'écocide au sein de la campagne internationale Stop Ecocide. Elle est Présidente d'Honneur de l'ONG Notre Affaire à Tous, ONG initiatrice de l'Affaire du siècle: premier recours contre l'inaction climatique de l'Etat français. Elle est aussi experte au sein de l'initiative des Nations Unies "Harmony with Nature" et membre du comité de direction de l'Alliance mondiale pour les Droits de la Nature (GARN). Elle a co-fondé en 2019 une école des Droits de la nature: Wild\&Legal. Elle a écrit deux ouvrages: Homo Natura, en harmonie avec le vivant (Buchet/Chastel, 2017), Un nouveau droit pour la Terre - Pour en finir avec l'écocide (Seuil, 2016).
}

Desenvolv. e Meio Ambiente usa uma Licença Creative Commons - Atribuição-NãoComercial-SemDerivações 4.0 Internacional 
barrages hydroélectriques (La Romaine). Puis en 2011, j'ai proposé bénévolement d'apporter un appui juridique durant la campagne du Cacique Raoni en Europe contre le barrage de Belo Monte. Dans les deux cas, j'ai d'une part pris conscience que le respect des droits des peuples autochtones étaient intimement liés au respect des droits de la nature, et d'autre part qu'en ne reconnaissant pas la nature sujet de droit, nous ne pouvions agir en justice qu'en réparation, après le dommage subi et seulement si des victimes humaines pouvaient être décomptées, et non en prévention par le biais de mesures conservatoires. De plus, force est de constater que la communauté internationale n'obtient pas d'accords suffisamment ambitieux ni contraignants souscrits entre États pour préserver le climat et la biodiversité et que parallèlement les règles du libreéchange tentent au contraire d'octroyer des droits supranationaux aux multinationales elles-mêmes visant à contourner les lois nationales et les cours de justice et les plaçant de fait au-dessus des États et donc des peuples. Les entreprises comme les humains sont des sujets de droit, les éléments de la nature ne sont que des choses et des propriétés. Cette vision anthropocentrée du droit nie nos liens d'interdépendance avec les non-humains et les systèmes écologiques de la Terre. Cela ouvre la porte à la prédation, la surexploitation et la pollution de tous les écosystèmes aquatiques et terrestres, et nous conduit face à un précipice, celui qui s'ouvre avec le changement climatique et la sixième extinction de masse en cours menaçant par ricochet les conditions de vie des générations futures. Ainsi pour pallier aux manquement du droit international actuel, il me semble nécessaire de procéder en deux étapes.

La première, qui est en cours, se fait à plusieurs niveaux. Il s'agit d'imposer un devoir de vigilance aux entreprises et de les soumettre à un régime d'obligations pour qu'elle respecte les droits humains et l'environnement. Face à l'émergence de ce droit, le secteur privé travaille ardemment avec l'ONU pour maintenir le caractère volontaire des directives basées sur la Responsabilité sociale des entreprises (RSE), qui ne sont pas conçues pour mettre fin ou punir les violations des droits de l'homme menées par les entreprises multinationales. Il faut aussi que le droit à un environnement sain soit reconnu de façon universelle, ce qui permettrait de poser le principe d'interdépendance au cœur de nos préoccupations actuelles, principe repris par l'initiative One Health ("une seule santé") qui promeut une approche intégrée, systémique et unifiée de la santé publique, animale et environnementale aux échelles locales, nationales et planétaire. Enfin il nous faudrait accepter d'assumer des devoirs vis-àvis des générations futures pour qu'ils héritent d'une planète habitable. Le droit à un environnement sain et à un climat stable doivent leur être reconnus, par là-même il s'agit donc de reconnaître les générations futures comme sujets de droit et de pouvoir les défendre en justice, par voie de représentation.

La seconde étape demande un changement de paradigme, une révolution culturelle, car elle nécessite de reconnaître comme pivot de notre système juridique le système Terre. Les rôles écologiques de ses écosystèmes, de ses espèces et de ses cycles bio-géochimiques doivent être protégés et leurs droits à exister, à se régénérer et à perdurer doivent être garantis. Ceci permettrait d'ester en justice au nom de la nature pour sa valeur intrinsèque et de renverser l'échelle des normes. Protéger l'habitabilité de la Terre, c'est protéger les droits fondamentaux humains à l'eau, à un air pur, à la santé, à l'alimentation. Les entités morales économiques, 
elles, devraient se mettre au service des peuples et du vivant, et non l'inverse. Et en ce qui concerne les dommages les plus sévères faits aux communs naturels, ceux dont les conséquences constituent une menace à la stabilité du Système Terre et à son habitabilité, ils devraient être poursuivis par une juridiction internationale telle que la Cour pénale internationale car ils menacent la Paix et la sécurité humaine de façon universelle, tout autant que les crimes internationaux les plus graves et déjà reconnus: les crimes contre l'humanité, les crimes de guerre, les génocides et les crimes d'agression. Étymologiquement, le terme d'écocide vient de Oikos en grec: la maison et occidere en latin: tuer. L'écocide est le fait de tuer notre maison commune, la seule que nous ayons, la Terre. L'urgence de la situation impose aujourd'hui de poser un cadre international répressif en mesure de poursuivre les auteurs des crimes les plus graves contre la biosphère, ses êtres vivants et les générations futures.

\section{De votre point de vue, quels sont les goulots} d'étranglement qui freinent les efforts d'inclusion de cette notion d'écocide dans le texte du Statut de Rome? Que faire pour qu'il soit enfin possible d'incriminer des États et des personnes juridiques et pas seulement des individus?

Le Statut de Rome, fondant la Cour pénale internationale, permet aujourd'hui de poursuivre des personnes physiques et donc de faire reconnaitre la responsabilité de décideurs et dirigeants, qu'ils soient politiques, économiques ou militaires; ce que ne permet pas, par exemple, la Cour de Justice internationale qui traite principalement des différends entre États mais aussi opposent parfois des États à des multinationales. En tant que citoyen, la plus haute Cour que vous pouvez saisir est la Cour pénale internationale. La difficulté pour faire évoluer le Statut de Rome, par exemple pour y inclure le crime d'écocide comme $5^{\text {e }}$ crime contre la paix et la sécurité humaine, est que cette décision appartient aux États membres, ceux qui ont ratifié le Statut. Un seul État volontaire peut porter une demande d'amendement à l'agenda d'une Assemblée générale des 123 États parties mais il faudra que $2 / 3$ des États parties votent en sa faveur pour qu'il soit retenu. La force de cette Assemblée est qu'elle est majoritairement constituée de pays victimes du changement climatique et que nombre d'entre eux sont dans l'attente d'une solution juridique contraignante. Au sein de la CPI, il n'y a pas de conseil de puissants ayant droit de véto comme cela est le cas au sein des Nations Unies. Certains États, qui ont influé sur le cours de l'histoire en demandant à ce que les crimes contre l'environnement ne soient pas retenus en temps de paix dans le projet de Code des crimes internationaux, sont fortement minoritaires aujourd'hui ou ne sont même pas signataires du Statut de Rome et n'ont donc plus leur mot à dire. C'est le cas des États-Unis par exemple.

L'autre difficulté est d'ordre conceptuel. Depuis des décennies, des juristes tentent de définir l'écocide. Il y a deux stratégies possibles: celle qui se veut pragmatique et celle qui se veut transformatrice. La pragmatique part du principe que la plupart des pays seront réticents à légiférer sur un crime qui conduirait à menacer leurs intérêts économiques. Elle considère donc que l'incrimination d'écocide ne doit reposer que sur des dommages étendus, durables et graves causés par des activités qui sont déjà illégales dans le droit international de l'environnement. Quid alors de la protection du climat sur laquelle 1'Accord de Paris n'est pas contraignant? 
Quid aussi de la protection des res communes et des res nullius, tous ces espaces naturels qui n'appartiennent à personne et qui sont exploitables par tous comme la Haute Mer, les Pôles etc.? Quid de la responsabilité de dirigeants politiques qui détruisent des forêts primaires sur leur sol constitutives de biomes transnationaux essentiels pour maintenir la biodiversité, le cycle des pluies, le production d'oxygène sur Terre?

La proposition transformatrice vise un objectif d'efficacité de l'arsenal pénal mit en œuvre, il s'agit bien de faire cesser la destruction des écosystèmes dont nous dépendons tous, humains et non-humains, pour survivre. Ce qui signifie faire cesser nombre d'activités qui les menacent, en faisant fi du principe de souveraineté nationale si nécessaire et en s'accordant sur un principe supérieur commun: la protection du système Terre. Il s'agit donc là de pousser, par l'instauration d'une période de transition entre l'adoption de l'amendement au Statut de Rome et son application, à une transformation de nos modèles de gouvernance, économiques et énergétiques, afin de retrouver une vie en harmonie avec la nature et garantir les conditions de vie des générations futures. Cette vision idéaliste s'attache à la gravité des conséquences quelle que soit l'activité qui en est la cause ou l'acteur. Elle ne s'embarrasse pas de conservatisme juridique et diplomatique, elle poursuit un objectif dicté par l'ampleur de la crise que nous traversons: garder une Terre habitable à la fin du siècle, ce qui devient de plus en plus compromis.

\section{Quel est le rôle de la reconnaissance des limi- tes planétaires dans l'établissement de normes juridiques universelles? Et dans un processus de transition écologique qui touche aux racines de la crise socioécologique globale?}

À l'échelle internationale, Ban Ki Moon, alors secrétaire général des Nations Unies, a évoqué, lors de l'Assemblée générale de 2011, les limites planétaires comme outil de mesure scientifique. S'adressant aux dirigeants du monde, il a déclaré: "Aidez-nous à défendre la science qui montre que nous déstabilisons notre climat et dépassons les limites planétaires à un degré périlleux" 3 . Le Groupe de haut niveau de l'ONU sur la viabilité du développement mondial (UN High-Level Panel on Global Sustainability) inclut alors la notion de limites planétaires dans son rapport de 2012 nommé "Pour l'avenir des hommes et de la planète: choisir la résilience"4.

Le changement climatique et l'érosion de la biosphère comportent, selon les scientifiques, des "limites fondamentales" qui interagissent entre elles. C'est le constat d'une équipe internationale de 26 chercheurs, menée par Johan Rockström du Stockholm Resilience Centre et Will Steffen de 1'Université nationale australienne, qui a identifiés

\footnotetext{
${ }^{3}$ United Nations News. Ban urges leaders to show greater commitment to agreement on climate change. UN News, 20 set. 2011. [En ligne] URL: <https://news.un.org/en/story/2011/09/387382>

${ }^{4}$ United Nations secretary-General's high-level panel on Global sustainability. Resilient People, Resilient Planet: A future worth choosing. New york: United Nations, 2012. [En ligne] URL: <https://en.unesco.org/system/files/GSP_Report_web_final.pdf>

${ }^{5}$ Steffen, W.; Richardson, K.; Rockström, J.; Cornellet, S.E. et al. Planetary boundaries: Guiding human development on a changing planet. Science, v. 347, n. 6223, 2015. doi: 10.1126/science.1259855
} 
dès 2009 neuf processus et systèmes régulant la stabilité et la résilience du système terrestre. Ensemble, ils fournissent les conditions d'existence dont dépendent nos sociétés. Ils ont ensuite déterminé les seuils à ne pas franchir pour chacun d'entre eux afin d'éviter de basculer dans un état planétaire inhospitalier et dangereux. Ainsi une grille de lecture est proposée concernant le taux d'émissions de $\mathrm{CO}_{2}$, le taux d'érosion de la biodiversité, mais aussi concernant la perturbation du cycle de l'azote et du phosphore, le seuil de déforestation, le taux d'acidification des océans, l'usage de l'eau douce. Sont aussi prises en considération les problématiques liées à l'appauvrissement de l'ozone stratosphérique, l'augmentation des aérosols dans l'atmosphère, la pollution chimique (composés radioactifs, métaux lourds, composés organiques synthétiques) dans la biosphère.

Le franchissement de chacune de ces limites nous conduit vers un "point de basculement" caractérisé à la fin par un processus d'extinction irréversible d'espèces et des conséquences catastrophiques pour l'humanité. Quand la biosphère est endommagée, son érosion a un impact sur le climat. La couverture végétale et le sol n'assument plus leur rôle crucial de régulation climatique directe, outre de stockage et de recyclage du carbone. La déforestation entraîne la disparition locale définitive des nuages et des pluies. La perte de plancton marin enraye la pompe à carbone qu'est l'océan.

Inscrire dans le droit le respect des limites planétaires permettrait aux institutions de l'État de cadrer les activités qui menacent le système Terre. La reconnaissance des limites planétaires comme normes juridiques permettrait précisément au légis- lateur mais aussi au juge d'apprécier la dangerosité d'une activité industrielle en s'appuyant sur les valeurs seuils déterminées par le Stockholm Resilience Center, et donc d'être en mesure d'apprécier si une activité industrielle est tolérable ou non. Que ce soit en droit pénal national ou international, il me semble que les limites planétaires peuvent constituer un très bon outil de mesure pour déterminer la gravité d'une atteinte portée à un écosystème; d'autant qu'il n'existe pas de consensus, depuis très longtemps, sur ce que l'on appelle en droit des "dommages étendus, graves et durables" à l'environnement. Il existe plusieurs définitions. Celle des Conventions de Genève diffère de celle de la Convention ENMOD sur les armes chimiques. Mais les Etats sont réticents à élever les limites planétaires au rang de normes car elles pourraient porter atteinte à la liberté d'entreprendre. J'ai évoqué le sujet à l'ONU à New York lors de mon intervention ${ }^{6}$ durant le Jour international de la Terre le 22 avril 2019. Il me semble en effet impératif, vu l'urgence écologique et climatique, de définir et respecter ce plafond écologique. On ne peut se contenter de l'empreinte écologique individuelle comme compas, car une fois de plus cela fait peser une responsabilité sur les citoyens tout en dédouanant les gouvernements, les politiques publiques et les industriels de leurs responsabilités face à l'écocide planétaire en cours. Et il y a urgence, à l'échelle globale nous avons déjà dépassé 4 des 9 limites planétaires, celles en lien avec le climat et la biodiversité - les plus fondamentales - mais aussi celles concernant la modification d'usage des sols (déforestation) et la perturbation de leurs cycles bio-géochimiques ( à cause de l'usage excessif d'engrais et des déjections issues de l'élevage).

\footnotetext{
${ }^{6}$ Disponible sur YouTube: <https://youtu.be/YJEfXmQNT3g>
} 
4. Comment participez-vous au débat contemporain autour du Principe de Précaution, outil jugé efficace pour contrecarrer les perspectives d'un possible collapse du métabolisme planétaire vers la fin de ce siècle?

La Déclaration de Rio (1992) définit le principe de précaution comme suit: "En cas de dommages graves ou irréversibles, l'absence de certitude scientifique absolue ne doit pas servir de prétexte pour remettre à plus tard l'adoption de mesures effectives visant à prévenir la dégradation de l'environnement".

Le principe de précaution, s'il devenait contraignant en droit international, permettrait de stopper des activités industrielles responsables d'écocides en cours ou susceptibles d'en provoquer, par le biais de mesures conservatoires. Une mesure conservatoire vise à conserver une ressource existante au moment de la conception d'un projet d'aménagement, dans le cadre des études d'impacts et des enquêtes publiques. De telles mesures sont aussi évoquées pour la compensation d'impacts socio-économiques ou sanitaires. Les citoyens pourraient alors saisir la justice dès les résultats d'une étude d'impact environnemental en phase de pré-projet industriel si un risque d'écocide semblait plausible. Le juge pourrait ainsi émettre, après investigation, des ordres de suspension de projets contribuant à transgresser les limites planétaires ou aux conséquences irréversibles en cas d'accident sur des communs planétaires ou des écosystèmes, tels que des projets de sites nucléaires, des projets de forage ou d'extraction de combustibles fossiles, des actions de déforestation massive ou des méthodes d'agriculture intensive. Mais pour que ce principe devienne une obligation, il faut obtenir un changement de paradigme concernant l'angle de l'instruction judiciaire en s'attachant à considérer la dangerosité d'une technologie au regard de l'étendue des conséquences en cas de catastrophe, et non au regard des risques potentiels. Prenons le risque nucléaire en exemple: le risque d'un "Fukushima" était minime, les conséquences de la catastrophe de Fukushima sont immenses. Si les études d'impact environnemental au moment du projet de construction du réacteur avaient été menées selon un critère de gravité plutôt qu'en lien avec la probabilité d'une catastrophe, cette centrale nucléaire n'aurait jamais vu le jour.

De façon générale, la reconnaissance croissante des principes de précaution et de pollueur-payeur, celle du droit de l'homme à un environnement sain et des droits des peuples autochtones, combinée aux efforts récents visant à conférer des droits aux écosystèmes, aux animaux et aux générations futures, sont des étapes dans la bonne direction. Mais tant que des responsables à la tête d'entités morales, telles que des États ou des multinationales, ne peuvent être inquiétés de poursuites pénales pour violations du droit de l'humanité à un environnement sain et/ou du droit de l'écosystème Terre à maintenir ses cycles et processus vitaux, alors il est très probable que nous n'obtiendrons pas de virage radical de notre modèle politique, économique et énergétique. La Terre continuera à être livrée aux appétits, aux dévastations et aux empoisonnements les plus meurtriers. Il nous faut donc créer une obligation de vigilance environnementale et sanitaire en droit pénal international et reconnaître comme criminelles toutes décisions qui auraient pour effet de menacer la sûreté de la planète. 
5. Face à la montée des impacts socioécologiques négatifs de l'industrie agroalimentaire hégémonique au niveau global - associés, entre autres, à la déforestation, au changement climatique, à la perte de biodiversité, à l'utilisation massive de pesticides, à la contamination des sols et des eaux - comment évaluez-vous le poids et la légitimité sociopolitique du modèle d'agrobusiness qui s'est imposé dans notre pays (et dans le Sud Global) dans les dernières décennies?

Dans la première partie de l'ouvrage "Un nouveau droit pour la Terre - Pour en finir avec l'écocide", je présente un inventaire des principales activités anthropiques considérées comme prédatrices, mettant en évidence les multiples impacts destructeurs générés par ces activités sur les systèmes vivants. L'industrie agroalimentaire est incluse dans cet inventaire.

L'élevage et l'agriculture industrielle sont parmi les principales causes de déforestation à l'échelle mondiale et contribuent au changement climatique de différentes manières, par exemple. $\mathrm{Au}$ niveau mondial, le secteur de l'élevage est responsable à lui seul de $14,5 \%$ des gaz à effet de serre anthropiques ${ }^{8}$, soit plus que les transports. La mécanisation de l'agriculture est en cause car elle emploie à grande échelle des énergies fossiles qui contribuent au réchauffement climatique. Mais les émissions de $\mathrm{CO} 2$ sont essentiellement dues à la déforestation par l'expansion des pâturages et des cultures fourragères pour le bétail. Les émissions de méthane proviennent essentiellement de la fermentation gastrique des ruminants et de la gestion des effluents d'élevage. Et enfin les émissions de protoxyde d'azote (qui a un potentiel de réchauffement climatique près de 300 fois plus élevé que le CO2) proviennent essentiellement des engrais, fumiers et lisiers. Au sein de l'élevage, les bovins sont les plus gros contributeurs de gaz à effet de serre, ils en émettent environ 6 à 7 fois plus que les cochons ou les poulets. Or nous consommons toujours plus de viande de bœuf et nous l'importons, en Europe par exemple, à moindre coût de forêts tropicales en danger, celles du Brésil en tête. Le Brésil possède le plus grand cheptel bovin au monde et est également le premier exportateur de viande bovine. Son cheptel est passé de 8,5 à 80 millions de têtes de 1974 à 2015 et il inonde le marché européen et américain?. La politique agricole et agraire brésilienne ellemême intègre l'ensemble des multiples facteurs qui influencent l'avancée de la déforestation ${ }^{10}$. La loi foncière, par exemple, prévoit que tout citoyen peut revendiquer la propriété d'une terre publique non affectée, y résider et la cultiver. Comme la plupart des titres de propriété restent attaquables si la terre n'est pas valorisée, les propriétaires sont poussés à défricher et à déboiser pour démontrer leur présence. De plus, en termes de spéculation foncière,

\footnotetext{
${ }^{7}$ Cabanes, V. Un nouveau droit pour la Terre. Pour en finir avec l'écocide. Paris: Éditions du Seuil, 2016.

${ }^{8} \mathrm{FAO}$ - Organisation pour l'alimentation et l'agriculture des Nations Unies. Elevage et changements climatiques - L'action de la FAO face au changement climatique/Elevage. FAO, 2016. [En ligne] URL: <http://www.fao.org/3/i6345f/i6345f.pdf>

${ }^{9}$ Selon les estimations de 1'Association brésilienne de l'industrie exportatrice de viande (Abiec): <http://abiec.com.br>

${ }^{10}$ LeTourneau, F.-M. Le Brésil maîtrise-t-il (enfin) la déforestation en Amazonie? Cybergeo: European Journal of Geography, Environnement, Nature, Paysage, doc. 753, 2015. doi: 10.4000/ cybergeo.27325
} 
une terre défrichée vaut plus qu'une terre boisée à la revente ${ }^{11}$. A ce tableau s'ajoutent les inégalités d'accès et de propriété foncière. La concentration des terres reste aujourd'hui un problème sérieux au Brésil: on estime que seulement $1 \%$ des propriétaires fonciers possèdent $45 \%$ des terres arables du pays ${ }^{12}$. Après l'essor du secteur agro-industriel, la situation est devenue si intolérable pour les petits agriculteurs que le gouvernement brésilien s'est vu contraint de mettre en place un système d'aide publique $^{13}$ (comme, par exemple, le Programme Fome Zero $^{14}$ ). Poussés par l'extension des plantations de canne à sucre, ils refluent vers l'Amazonie où ils défrichent pour planter. Mais pour l'instant, ce sont d'abord les grands propriétaires qui transforment le nord de l'Amazonie en un vaste pâturage. On estime que $83 \%$ de la croissance du cheptel bovin du Brésil de 1990 à 2007 a été réalisée en Amazonie $^{15}$ et l'élevage y est très majoritairement extensif. Selon 1'Institut National de Recherches spatiales (INPE) ${ }^{16}$ brésilien qui analyse les images satellites, en douze mois 11.088 kilomètres carrés de forêt ont été détruits dans 1'Amazonie (entre août 2019 et juillet 2020). Ce bilan de destruction massive correspond à une zone déboisée plus large que la Jamaïque. Toujours selon le même institut, sur les neuf États qui composent l'Amazonie légale brésilienne, quatre d'entre eux (Pará, Mato Grosso, Amazonas et Rondônia) représentaient ensemble $87,8 \%$ de la déforestation estimée en 2020. Et si l'on considère l'ensemble des données de l'INPE depuis 1988 (lorsque des mesures plus précises ont commencé à être prises au Brésil) jusqu'en 2020, on estime que plus de 813.000 kilomètres carrés ont été déjà déboisés dans l'Amazonie légale.

L'élevage en Amazonie a d'autres conséquences. La viande doit être acheminée vers le sud du pays et vers la mer. Pour relier le sud au nord, un plan d'asphaltage de la route BR-163 a été lancé, ainsi que des projets de transport fluvial par barges à grande capacité, afin d'accéder à la mer via l'Amazone. Toute nouvelle infrastructure devient un facteur aggravant de déforestation, car chaque grande route, chaque port créés incite à la conquête de nouveaux territoires ${ }^{17}$. Les forestiers arrivent les premiers pour couper et récupérer les bois nobles. Ils construisent eux-mêmes des routes pour pénétrer dans le couvert forestier et certains ne se gênent pas pour pratiquer des coupes illégales en territoire indigène. Concernant le Sud de l'Amazonie, il est le

\footnotetext{
${ }^{11}$ LeTourneau, F.-M. Le Brésil maîtrise-t-il (enfin) la déforestation en Amazonie? Cybergeo: European Journal of Geography, Environnement, Nature, Paysage, doc. 753, 2015. doi: 10.4000/ cybergeo. 27325

${ }^{12}$ IBGE - Institut Brésilien de Géographie et de Statistique. Recensement agricole 2017. [En ligne] URL: <https://censoagro2017.ibge.gov.br/ templates/censo_agro/resultadosagro/index.html>

${ }^{13}$ Cabanes, V. Un nouveau droit pour la Terre. Pour en finir avec l'écocide. Paris: Éditions du Seuil, 2016.

${ }^{14}$ Silva, J.G.da; Del Grossi, M.E.; França, C.G.de (Orgs.). Fome Zero (Programa Hambre Cero): La experiencia brasileiña. Brasília: MDA/ FAO, 2012. [En ligne] URL: <http://www.fao.org/3/i3023s/i3023s.pdf>

${ }^{15}$ LeTourneau, F.-M. Le Brésil maîtrise-t-il (enfin) la déforestation en Amazonie? Cybergeo: European Journal of Geography, Environnement, Nature, Paysage, doc. 753, 2015. doi: 10.4000/ cybergeo. 27325.

${ }^{16}$ INPE - Institut National de Recherches Spatiales. Note Technique - Estimation PRODES 2020. [En ligne] URL: <http://www.obt.inpe.br/ OBT/noticias-obt-inpe/estimativa-de-desmatamento-por-corte-raso-na-amazonia-legal-para-2020-e-de-11-088-km2/NotaTecnica_Estimativa_PRODES_2020.pdf>.

${ }^{17}$ LeTourneau, F.-M. Le Brésil maîtrise-t-il (enfin) la déforestation en Amazonie? Cybergeo: European Journal of Geography, Environnement, Nature, Paysage, doc. 753, 2015. doi: 10.4000/ cybergeo. 27325
} 
théâtre d'une agriculture extensive en monoculture, autre facteur responsable de la déforestation et participant de multiples manières au réchauffement climatique. Certaines exploitations peuvent défricher plus de 5.000 hectares en une année pour y planter du soja, du sucre, du maïs ou du coton ${ }^{18}$. Le soja, transgénique à $92 \%$, sert à produire des tourteaux pour le bétail à l'étranger mais aussi à nourrir les $10 \%$ de bovins brésiliens qui sont élevés de façon intensive une partie de leur vie, c'est-à-dire confinés dans d'immenses étables ${ }^{19}$. La culture extensive de soja est ainsi devenu le second marqueur de la conquête agricole amazonienne. Comme il constitue la base de la fabrication de tourteaux pour l'élevage, sa culture suit la croissance de la consommation de viande dont le pays a tiré profit au niveaux national et international en développant ce marché pour $l^{\prime}$ 'export ${ }^{20}$. Pour nourrir son propre bétail, l'Europe consomme en effet du soja en grande quantité importé depuis le Brésil ou l'Argentine, et la France en est d'ailleurs le premier destinataire européen ${ }^{21}$. Le soja est aussi prisé pour la production d'huile végétale alimentaire en Asie principalement. La croissance de la production du soja au Brésil a ainsi augmenté de $40 \%$ entre 2008 et $2014^{22}$ et le pays est devenu le premier exportateur mondial, devant les États-Unis et l'Argentine. Concernant la production de sucre au Brésil, elle est destinée à la production d'agro-carburants à base d'éthanol. À eux seuls, les États-Unis et le Brésil produisent 70\% de l'éthanol mondial ${ }^{23}$. Si ces monocultures sont une source de déforestation massive, il faut savoir qu'elles appauvrissent en plus les populations locales qui trouvent de moins en moins de produits vivriers sur le marché local et sont contraints de les importer et de les payer au prix fort. De plus, l'ammoniac dégagé dans l'atmosphère par cette agriculture contribuent au phénomène des pluies acides. Les arbres qui reçoivent de l'eau de pluie acide voient leurs feuilles tomber et ils se nécrosent. Ces pluies acides impactent aussi gravement les milieux aquatiques en tuant la vie qu'ils abritent. Elles participent à l'eutrophisation des sols par un apport excédentaire d'azote dans les milieux naturels, ce qui conduit à une réduction de la biodiversité. L'usage des insecticides est un facteur aggravant, car ces biocides contaminent les écosystèmes et empoisonnent les populations (humaines et non humaines) qui en font partie. Les impacts des pesticides sur les espèces pollinisatrices, notamment les abeilles et les papillons, dans le contexte actuel d'accélération de la sixième extinction de masse sont particulièrement inquiétants. Les récentes recherches axées sur les insecticides à base de néonicotinoïdes témoignent d'effets létaux et sublétaux sur les abeilles ${ }^{24}$. Les pollinisateurs sont indispensables à la survie et à

\footnotetext{
${ }^{18}$ Cabanes, V. Un nouveau droit pour la Terre. Pour en finir avec l'écocide. Paris: Éditions du Seuil, 2016.

${ }^{19}$ Cabanes, V. Un nouveau droit pour la Terre. Pour en finir avec l'écocide. Paris: Éditions du Seuil, 2016.

${ }^{20}$ Greenpeace France. Mordue de viande - L'Europe alimente la crise climatique par son addiction au soja. Paris: Greenpeace, 2019. [En ligne] URL: <https://cdn.greenpeace.fr/site/uploads/2019/06/hooked_on_meat_FR_web.pdf $>$.

${ }^{21}$ Voir Planétoscope - Consommation de soja en France: $<$ https://www.planetoscope.com/cereales/1713-consommation-de-soja-en-france.html> ${ }^{22}$ Voir Planétoscope - Production mondiale de soja: <https://www.planetoscope.com/cereales/200-production-mondiale-de-soja.html>

${ }^{23}$ Voir BP - Renewable energy: <https://www.bp.com/en/global/corporate/energy-economics/statistical-review-of-world-energy/renewable-energy.html>

${ }^{24}$ Rundlöf, M.; Andersson, G.; Bommarco, R. et al. Seed coating with a neonicotinoid insecticide negatively affects wild bees. Nature, 521,77-80, 2015. doi: 10.1038 /nature 14420
} 
l'évolution de $80 \%$ des plantes à fleurs, qu'elles soient cultivées ou non. Sans eux, la diversité génétique au sein des espèces déclinerait, et, à terme, les espèces elles-mêmes seraient en péril, la seule action du vent ne suffisant généralement pas à la reproduction sexuée. On considère que $35 \%$ des ressources alimentaires mondiales sont dépendantes des insectes pollinisateurs, il en va donc aussi de la survie de l'humanité.

\section{Dans quelle mesure le Tribunal Monsanto} (tenu à La Haye entre 2016 et 2017) a-t-il été important pour mettre en évidence la responsabilité des entreprises agrochimiques sur la dégradation des écosystèmes et des modes de vie, ainsi que pour faire avancer l'élaboration et la légitimation du concept d'écocide?

Le Tribunal Monsanto est un procès citoyen, sans reconnaissance officielle, dont le but est d'alerter l'opinion et de faire avancer le droit. Durant deux jours du 15 au 16 octobre 2016 à La Haye, cinq magistrats professionnels (venant d'Argentine, de Belgique, du Canada, du Mexique et du Sénégal) ont auditionné une trentaine de témoins, d'experts, de victimes, d'avocats face à la mise en accusation de Monsanto devenu Bayer. L'avis consultatif des juges ${ }^{25}$ rendu en avril 2017 ne laisse place à aucun doute quant aux agissements de Monsanto, et cette opinion juridique peut servir de jurisprudence. Le tribunal estime que la multinationale contrevient aux réglementations et au respect des droits fondamentaux par "des pratiques qui ont de graves répercussions sur l'environnement" et qui affectent les droits des peuples autochtones et des communautés locales. Il considère aussi que l'entreprise bafoue les droits à l'alimentation et à la santé. Le tribunal détaille en particulier "la commercialisation agressive de semences OGM" qui altère ces droits "en forçant des agriculteurs à adopter des modes de culture qui ne respectent pas les pratiques des cultures traditionnelles". Les cinq juges dénoncent aussi les pratiques de Monsanto qui nuisent à la liberté de la recherche scientifique, ainsi qu'à "la liberté d'expression et au droit à l'accès à l'information". Enfin le Tribunal a estimé "que le temps est venu de proposer la création d'un nouveau concept juridique pour le crime d'écocide et de l'intégrer dans une future version amendée du statut de Rome établissant la Cour pénale internationale". Le Tribunal a reconnu que Monsanto pourrait être poursuivi si ce crime était intégré dans le droit pénal international. Selon la Présidente du Tribunal, la juge Françoise Tulkens, cette opinion juridique devrait permettre d'asseoir le nouveau crime d'écocide et d'aider les États à mieux faire respecter les droits fondamentaux que sont l'alimentation, la santé, l'information, le droit à un environnement sain. Il s'agit aussi d'inciter les victimes à utiliser les points juridiques pour poursuivre Monsanto/ Bayer devant les tribunaux nationaux. Le droit des entreprises, des règles du commerce mondial, sont en train de primer sur les droits de l'homme et ceux de la nature. Il est temps de redéfinir la hiérarchie des normes et d'être en capacité de pouvoir poursuivre des acteurs économiques en justice, personnes physiques et entités morales, quand ils menacent l'habitabilité de la Terre.

\footnotetext{
${ }^{25}$ Résumé de l'avis juridique consultatifémis par les juges du Tribunal Monsanto: $<$ https://fr.monsantotribunal.org/upload/asset_cache/119865256. pdf $>$
} 
7. Quelle est votre lecture de l'émergence de la pandémie de Covid-19 dans ce contexte de complexification et d'accélération de la crise planétaire du nexus écologie \& développement?

Cette crise nous révèle notre vulnérabilité et nos liens d'interdépendance avec le reste du vivant. Le Covid est une manifestation de l'écocide en cours. Une zoonose, c'est-à-dire une maladie qui se transmet d'un animal sauvage vertébré à un humain, ne peut se transmettre que quand les humains sont trop proches des espaces sauvages. La déforestation y contribue, tout comme l'urbanisation galopante. L'épidémie de Covid-19 est un exemple, selon les chercheurs de l'IPBES ${ }^{26}$, de la manière dont l'érosion de la biodiversité et la destruction des habitats naturels peuvent menacer l'humanité. Une étude, publiée le 1er juin 2020 dans la revue Proceedings of the National Academy of Sciences ${ }^{27}$, explique que la sixième extinction de masse s'accélère et met en péril la survie de l'espèce humaine elle-même. "Lorsque l'humanité extermine d'autres créatures, elle coupe la branche sur laquelle elle est assise, détruisant des parties essentielles du système qui permet le maintien de la vie sur Terre", explique Paul Ehrlich, professeur à l'Université de Stanford, un des co-auteurs de l'étude.

La Covid a aussi mis en lumière les inégalités sociales. On constate que ce sont les populations les plus pauvres qui sont les plus touchées. Les malades chroniques victimes d'obésité développent aussi du diabète, de l'hypertension, des cancers et ils ont bien davantage succombé à la Covid-19. L'obésité est souvent liée à des facteurs environnementaux, à une exposition aux perturbateurs endocriniens et aux pesticides, mais aussi à la pollution atmosphérique et à l'alimentation ultra transformée. Or cette exposition est elle-même liée à leur condition sociale qui les conduit à acheter une nourriture peu chère et hautement calorique mais aussi les oblige à vivre dans des environnements plus pollués.

La question qui demeure, avec la récession économique qui se profile, concerne les choix qui seront faits par les gouvernements partout dans le monde en termes de relance. Organiseront ils une relance qui prend en considération le risque climatique, écologique, profitant de cette crise pour repenser complètement le système? J'en doute mais j’y aspire. Il faudrait, dans la lignée de la théorie du beignet de Kate Raworth ${ }^{28}$ - the Donut theory - que nous profitions de cette crise pour reconstruire une société qui garantisse les besoins fondamentaux de chacun.e, autrement dit un "plancher social" tout en acceptant, et c'est cela la nouveauté, de fonctionner avec un "plafond écologique" déterminé par les "limites planétaires", pierre angulaire de mon plaidoyer. Nous devons rompre avec l'obsession de la croissance qui est incompatible avec le principe de finitude propre à la vie. Cette idéologie économique nous conduit vers un avenir mortifère.

\footnotetext{
${ }^{26}$ IPBES - Intergovernmental Science-Policy Platform on Biodiversity and Ecosystem Services. Pandemic Reports - Escaping the 'Era of Pandemics', 2020. [En ligne] URL: <https://www.ipbes.net/pandemics>

${ }^{27}$ Ceballos, G.; Ehrlich, P.R.; Raven, P.H. Vertebrates on the brink as indicators of biological annihilation and the sixth mass extinction. Proceedings of the National Academy of Sciences, 117(24), 13596-13602, 2020. doi:10.1073/pnas.1922686117

${ }^{28}$ Kate Raworth exploring doughnut economics: <https://www.kateraworth.com/doughnut/>
} 
8. Enfin, dans un contexte d'émergence de gouvernements autoritaires, quelles seraient vos recommandations aux citoyens et aux groupes sociaux engagés dans le contrôle social du processus de marchandisation du monde qui avance à l'ombre de l'ordre juridique dominant - à visée anthropocentrique?

L'économie doit revenir à son sens étymologique premier: la bonne gestion de la maison commune. Nous prenons conscience que nous ne sommes pas maitres de la nature, que nous sommes interconnectés et que les règles du vivre-ensemble doivent maintenant prendre en considération les non-humains. Il y a une concordance de différents mouvements qui aspirent à un autre modèle de société, où pourrait entrer dans le débat démocratique des entités naturelles dont le rôle écologique est vital pour maintenir la sûreté de la planète pour tous. Face à des régimes de plus en plus autoritaires, je constate aussi que le "monde d'après" est en train de se construire, en parallèle, à l'échelle locale. Il y a des réseaux de solidarité extraordinaires qui se sont mis en place pendant le confinement, pour penser et mettre en œuvre une résilience, une souveraineté alimentaire, une économie relocalisée et une solidarité en action. Mais tant que l'État en tant qu'institution existera, il aura à charge d'être garant du respect global du plancher social et du plafond écologique. Il est donc très important que la société civile s'empare de toutes ces idées et continue à faire pression sur les gouvernements et les élus. Justice économique, sociale, climatique et environnementale sont indissociables. C'est pourquoi dans le monde entier, des citoyen(ne)s et des associations se tournent vers les tribunaux pour faire respecter leurs droits et ceux de la nature. Ils contestent le manque d'ambition des politiques des États et engagent la responsabilité des entreprises les plus polluantes. C'est ce que nous avons fait en créant l'association Notre Affaire à Tous ${ }^{28}$ en France en 2015, qui poursuit l'État français en justice pour non-respect de ses obligations climatiques ${ }^{29}$ et qui poursuit aussi la multinationale pétrolière Total en justice ou le groupe Casino (qui contribue à la déforestation en Amazonie) pour manquement à leur devoir de vigilance ${ }^{30}$. Ceci en attendant de pouvoir se tourner vers une juridiction internationale qui reconnaîtrait le crime d'écocide.

\section{Remerciements}

Les organisateurs remercient, encore une fois, la sollicitude et l'ouverture au dialogue de Valérie Cabanes, qui a offert, dans son témoignage dense, une précieuse contribution à l'édition spéciale "L'agrobusiness en temps d'effondrement planétaire: approches critiques".

\footnotetext{
${ }_{28}$ Association Notre Affaire à Tous: <https://notreaffaireatous.org >

${ }^{29}$ Notre Affaire à Tous - L'Affaire du Siècle: <https://notreaffaireatous.org/actions/laffaire-du-siecle/>

${ }^{30}$ Notre Affaire à Tous - Multinationales <https://notreaffaireatous.org/actions/polleurs-payeurs/>
} 